\title{
Performance Analysis of MANET Routing Protocols using different Mobility Models and Varying Speed
}

\author{
Prof. S. S. Asole ${ }^{1},{ }^{2}$ Dr. P. M. Jawandhiya \\ ${ }^{1}$ Associate Professor, Department Of CSE, B.N.C.O.E., Pusad, India. \\ ${ }^{2}$ Principal, PLIT, Buldana India.
}

\begin{abstract}
A mobile ad-hoc network is a continuously self-configuring, infrastructure less, network of mobile devices. It is the network of mobile nodes without any fixed topology. In this network, each node acts as both hosts and router simultaneously. Each device in a MANET is free to move independently in any direction, and will therefore change its link to other devices frequently. In such a network there are lots of possibilities of mobility of nodes. Therefore mobility of nodes is challenging issue for designer. We have surveyed comparative performance analysis of different routing protocols. Under the different mobility models in term of Packet Delivery Ratio, Average End to End Delay. We have carefully studied the behavior of these routing protocols and compared their performance in different scenarios based. This paper defines which protocol to use in different cases such as if time is important or if data is important in various mobility modes such as all node mobility, destination node mobility and source node mobility and proposed the new algorithm on the basis of protocols evaluation in different situation.
\end{abstract}

\section{INTRODUCTION}

Nodes in the network may be able to communicate directly with each other, for example when they are within wireless transmission range of each other. However, ad hoc networks must also support communication between nodes that are indirectly connected by a series of wireless hops through other nodes. Routing is well studied feature of such networks because mobile nodes may move in various directions, which can cause existing link to break and the establishment of new routes.

A good routing protocol should minimize the computing load on the host as well as the traffic overhead on the network. There are three types of routing protocol in Mobile Ad-Hoc Networks Proactive (Table Driven) routing protocol, Reactive (Demand Driven) routing protocol and Hybrid Routing Protocol[2]. Routing protocol is use to find the route between communication nodes. A mobility model is representing the movement behavior of mobile node.

\section{EXISTING WORK}

\subsection{MANET Routing Protocols}

Proactive routing protocol provide a fast response to topology change continuously monitoring topology change and disseminating the related information as needed over the network. However rapid response to topology changes is the increase in routing overhead, and this can lead to smaller packet delivery ratio and longer delay when topology changes increase. Reactive routing protocols form a route if needed and reduce the routing overhead. However the long setup time in route discovery and slow response to route changes can offset the benefit derived from on demand and lead inferior performance.

\subsubsection{Table-driven protocols}

These protocols have their ability to maintain routing tables that store information regarding the routes from one node in the network to the rest of other nodes. Here, all nodes update their tables to preserve compatibility by exchanging routing information between the participating nodes. When the topology of the network changes, the nodes distribute update messages across the network. These protocols may be easy to implement, but the major limitation is that, due to the inherently highly mobile and dynamic nature of ad-hoc networks, the maintenance of routing information in these tables is challenging.

\section{- DSDV (Destination-Sequenced Distance -Vector}

\section{Routing Protocol)}

This protocol is based on the Bellman-Ford classical routing mechanism. Here each mobile node maintains a routing table that includes all accessible destinations, the number of hops necessary for reaching that destination and the sequence of the digits appropriate to that destination. Routing table entries are tagged with sequence of digits which are originated by the destination nodes. This sequence of digits is used to distinguish new routes from old routes and also to determine the creation of a ring. Route updates are transmitted either periodically or immediately after a significant topology change is being detected. DSDV protocol generates a supplementary traffic that adds to the real data traffic.

\subsubsection{Reactive (On Demand) Routing Protocol}

These types of routing protocols create routes only when desired by the source node. When a source node 


\section{Available online at www.ijrat.org}

requires route it initiates a route discovery process to find the route to the destination. This type of protocols find route by flooding the network with route request packet. This process is completed once a route is found or all possible route permutations have been examined. Once a route has been established, it is maintain by a route maintain procedure. Then this route is used for further communication.

AODV (Ad-Hoc On Demand Distance Vector Routing)

AODV does not attempt to maintain routes from every node to every other node in the network. It only require route when necessary and do not need maintain route that are not use currently. When a source node need route to certain destination, It broadcast a route request (RREQ) packet to all other neighbors. This packet contains a IP address and sequence number as well as the destination IP address and last known sequence number. The RREQ also contain the broadcast ID. When a node receives a RREQ it first checks IP address and broadcast ID. If it has already seen a RREQ with same IP address and broadcast ID then discard the packet otherwise rebroadcast the packet. Once the RREQ reaches the destination node respond by unicast a route reply (RREP) to the source. This route is use for communication between sources to destination.

\section{DSR (Dynamic Source Routing)}

The DSR is an on-demand routing protocol. The DSR routing protocol consists of two major phase's route discovery and route maintenance. When a source nose $\mathrm{S}$ want to sand a packet to destination D. It establish a route from $\mathrm{S}$ to $\mathrm{D}$ this phase is called route discovery. Route discovery use only when no route between source to destination. Second phase route maintenance phase requires in case of route failure, it involve another route to destination. Then the source $\mathrm{S}$ can be use an alternate route to destination $\mathrm{D}$, if it known one, or invoke route discovery.

\subsection{Mobility Models}

Mobility Models describes how speed, acceleration and direction of the node change over time .

\subsubsection{Random Waypoint Mobility Model}

The Random Waypoint Mobility Model is the most common mobility model used in MANET researches. The RWP model is random model for movement of mobile use, how their location, velocity and acceleration change over time. A mobile node begins the simulation by waiting a specified pause time. After this pause time it selects a random direction and random speed between $0 \mathrm{~m} / \mathrm{sec}$ to $\mathrm{Vmax} \mathrm{m} / \mathrm{sec}$ in the network area. After reaching this destination node wait again pause time and then select a new direction and speed for movement . The node keeps moving until reaches its direction at that speed. If a node selects a far destination and low speed travels for a long time. AODV performs better than DSR and DSDV in random waypoint mobility model [1]. AODV gives the better packet delivery ratio[1]. The overall performance of AODV is better in random waypoint mobility model[1].

\subsubsection{Random Direction Mobility Model}

In Random Direction the mobile node select a direction travel to the border of the network area. On reaching the boundary it wait for a specific pause time and then

choose the new direction to follow. This model does not suffer from the density waves in the centre of the simulation space that Random Waypoint Model does. For the Random Waypoint Mobility Model the probability of choosing a location near center is very high.

\subsubsection{Random Walk Mobility Model}

It is a simple mobility model based on random direction and speed. In this mobility model mobile node choose a random direction and speed for movement. The new direction and speed choose from predefined ranges. On reaching the boundary of simulation area the node reflect back with an angle determined by incoming direction.

\section{SYSTEM IMPLEMENTATION AND DISCUSSION}

\subsection{Performance Metrics}

To evaluate the performance of AODV, DSR, DSDV routing protocols two performance metrics Packet Delivery Ratio(PDR) Average end to end delay[3] by varying the speed of mobile nodes are considered

A. Packet Delivery Ratio $\mathrm{PDR}=($ packet receives $/$ packet sent $)$

B. Average End to End delay

It includes delay caused by latency, buffering, queuing, transmission and route discovery

The given table 1 represents the Evaluation of protocols in different mobility model with varying speed of nodes [1] 


\section{Available online at www.ijrat.org}

\begin{tabular}{|l|l|c|c|c|}
\hline Parameter & Protocol & $\begin{array}{l}\text { Random } \\
\text { Waypoint }\end{array}$ & $\begin{array}{l}\text { Random } \\
\text { Walk }\end{array}$ & $\begin{array}{c}\text { Random } \\
\text { Direction }\end{array}$ \\
\hline \multirow{4}{*}{$\begin{array}{l}\text { Packet } \\
\text { Delivery } \\
\text { Ratio }\end{array}$} & AODV & $\begin{array}{c}\text { High } \\
\text { (highest) }\end{array}$ & $\begin{array}{c}\text { Highest } \\
\text { (highest) }\end{array}$ & $\begin{array}{c}\text { Highest } \\
\text { (highest) }\end{array}$ \\
\cline { 2 - 5 } & DSR & $\begin{array}{c}\text { Highest } \\
\text { (high) }\end{array}$ & $\begin{array}{c}\text { High } \\
\text { (high) }\end{array}$ & $\begin{array}{c}\text { High } \\
\text { (high) }\end{array}$ \\
\hline (low) & $\begin{array}{c}\text { Low } \\
\text { (low) }\end{array}$ & $\begin{array}{c}\text { Low } \\
\text { (low) }\end{array}$ \\
\hline \multirow{2}{*}{$\begin{array}{l}\text { Average } \\
\text { end to end } \\
\text { Delay }\end{array}$} & AODV & $\begin{array}{c}\text { High } \\
\text { (high) }\end{array}$ & $\begin{array}{c}\text { Highest } \\
\text { (highest) }\end{array}$ & $\begin{array}{c}\text { Highest } \\
\text { (highest) }\end{array}$ \\
\cline { 2 - 5 } & DSR & $\begin{array}{c}\text { Low } \\
\text { (low) }\end{array}$ & $\begin{array}{c}\text { High } \\
\text { (low) }\end{array}$ & $\begin{array}{c}\text { Low } \\
\text { (low) }\end{array}$ \\
\cline { 2 - 5 } & DSDV & $\begin{array}{c}\text { Highest } \\
\text { (highest) }\end{array}$ & $\begin{array}{c}\text { Low } \\
\text { (high) }\end{array}$ & $\begin{array}{c}\text { Low } \\
\text { (high) }\end{array}$ \\
\hline
\end{tabular}

Table1: Comparison of AODV, DSR and DSDV Protocol using Different Mobility Models.

\section{Packet Delivery Ratio}

- In all three protocols PDR is decreases linearly with increasing the speed of nodes[1].

- PDR for reactive routing protocols AODV and DSR were better than the proactive DSDV[1].

- From two reactive routing protocols AODV and DSR, AODV gives slightly more PDR than DSR[1].

\section{A. Average end to end delay}

- For all three protocols average end to end delay increases as the speed of node increases[1].

- In Random Waypoint mobility model end to end delay is high for DSDV and low for DSR[1]

- In Random Walk mobility model end to end delay is high for AODV and low for DSDV up to certain limit of speed then it increases and lowest end to end delay is given by DSR[1].

- In Random Direction mobility model end to end delay is high for AODV and low for DSR[1].

- AODV performs better than DSR and DSDV in Random waypoint mobility model[1].

- In random waypoint and random direction as the speed of node increases the packet delivery ratio of DSR decreases with high degree respect to the AODV and DSDV[1].

- The overall performance of AODV is better in random waypoint mobility model[1].

- In the random walk and random direction models the end to end delay is very high for AODV than DSR and DSDV protocols. If the speed of node is $10 \mathrm{~m} / \mathrm{sec}$ then the AODV perform better but if we increase the speed up to $40 \mathrm{~m} / \mathrm{sec}$ performance of AODV decrease because end to end delay is very high[1].

- The packet delivery ratio is high of AODV in random walk and random direction, but the end to end delay was also very high for AODV protocol[1].
So the overall performance of DSR is better than the AODV and DSDV in random walk and random direction mobility model[1].

\section{PROPOSED WORK}

Sometimes it is important to deliver data from source to destination in time in such a case some form of data loss is tolerable and many time it is important to deliver data without any loss but some form of time delay not matters in such a cases. Putting such conditions in front, study of two protocols DSR and DSDV gives solution for such cases. The metrics Packet Delivery Ratio and End to End Delay are important here. More packet delivery ratio means less packet lost during transmission and end to end delay indicate total delay for packet.

\section{Proposed Algorithm-}

NON : 10

Protocol: DSR, DSDV

Parameter: throughput, avg end to end delay

Movement: dest node, source node, all nodes

If movement of all nodes

$$
\begin{aligned}
& \text { If data is imp } \\
& \text { Prefer DSDV } \\
& \text { Else }
\end{aligned}
$$

$$
\text { Prefer DSR }
$$

Else

If movement of dest node only

If data is imp

$$
\text { Prefer DSDV }
$$

Else

$$
\text { Prefer DSR }
$$

Else

If movement of source node only Prefer DSDV

\section{Description -}

Here network of 10 nodes is considered. In the network one node is source node, one is destination node and all other nodes are intermediate nodes act as a router in MANET to deliver the data from source node to destination node. Protocols taken are DSR and DSDV. For evaluation purpose performance metrics such as throughput and average end to end delay are considered. In such case three mobility modes are possible. First mode is when only source node in the network is mobile. In second mode only destination mode in the network is mobile. In third mode all the nodes of the network are mobile.

\section{Impacts of these modes on the performance of} protocols are as follows.

When all the nodes in the network are mobile then it is DSDV gives high packet delivery ratio than DSR, but average end to end delay is less in DSR. So, it is 


\section{Available online at www.ijrat.org}

concluded that for user if data is important than time then it is good to use DSDV. If time is important then use DSR. If destination node in the network is mobile and all other are non-mobile then DSDV gives more packet delivery ratio than DSR but average end to end delay is less in DSR. So, if data is important use DSDV otherwise DSR. If source node in the network is mobile then DSR is of no use.

\section{CONCLUSION AND FUTURE WORK}

This paper determines which protocol to use in different cases. When Data is important and all the nodes in the network are mobile then use DSDV otherwise if time is important factor then use DSR. When data is important and only destination node is mobile then use DSDV otherwise if time is important then use DSR. If source node only is mobile then use only DSDV. The proposed algorithm is very useful for researcher to check the performance of different protocols under different mobility models without checking each case using NS2.

Future work will be to evaluate the performance of various new protocols or modified version of these protocols could also be compared.

\section{Acknowledgements}

I have inadequate words to express my deep sense of gratitude towards my guide Dr.P.M.Jawandhiya who was a constant source of inspiration, encouragement and help throughout the process of preparing the paper and without whom continuous guidance this work would not have been possible.

I also extend my sincere thanks to Principal Dr. H. B. Nanvala for his encouragement, providing the adequate facilities and for providing an outstanding academic environment.

\section{REFERENCES}

[1] Prateek Chudhary, Viomesh Singh, "Performance Evaluation of mobile Ad-Hoc Networks Routing Protocol AODV,DSR and DSDV in different mobility models with varying speed of nodes", International Journal of Computer Science and Information Technologies(IJCSIT), Vol.5 (3), 2014

[2] K. Natarajan, Dr. G. Mahadevan, "A succinct Comparative Analysis and Performance Evaluation of MANET Routing Protocols", 2013

[3] Hemant Rai "A Comparison of Performance Metrics for Various Routing Protocols in MANET", 2014

[4] Muhammad Asif Khan, Sahibzada Zakiuddin, "Research Review of Development of Novel Routing Algorithms for Mobile Ad-hoc Networks", 2013

[5] Marco Conti, Silvia Giordano "Mobile Ad Hoc Networking: Milestones, Challenges, and New Research Directions", 2014
[6] Dr.P.M.Jawandhiya, S.S.Asole ,'Performance analysis of Mobil;ity Models using Routing Protocols, Traffic Models and Provides an outcome as to which routing protocol is better", 2nd IEEE International conference on Recent Adavaces and Innovations in Engineering (ICRAIE),2016. 